Article

\title{
Adult and Pediatric Intra-Institutional Trends of Ciprofloxacin Susceptibility in E. coli Positive Urinary Cultures
}

\author{
Winifred Owumi ${ }^{1}$, Niaz Banaei ${ }^{2}$ and Linda D. Shortliffe ${ }^{1, *}$ \\ 1 Department of Urology, Stanford University Medical Center, 750 Welch Rd, MC: 5725, \\ Stanford, CA 94305-5725, USA; E-Mail: isi@stanford.edu \\ 2 Departments of Pathology and Medicine, Stanford University Medical Center, 300 Pasteur Drive, \\ Stanford, CA 94304, USA; E-Mail: niazbanaei@stanford.edu \\ * Author to whom correspondence should be addressed; E-Mail: lindashortliffe@stanford.edu; \\ Tel.: +1-650-498-5042; Fax: +1-650-723-4055.
}

Received: 13 February 2014; in revised form: 3 April 2014 / Accepted: 4 April 2014 /

Published: 21 April 2014

\begin{abstract}
Antimicrobial drug resistance in treatment of urinary tract infection (UTI) continues to rise worldwide. To examine contributions of physician prescribing patterns to fluoroquinolone (ciprofloxacin, CP) resistance, we examined Escherichia coli (E. coli) resistance patterns in urinary cultures. Since CP usage is limited in children, we compared CP resistance trends in adults and children to those of more commonly used trimethoprim-sulfamethoxazole (TMP-SMX) and nitrofurantoin (NF). Our data show that although the general pediatric population has lower resistance to ciprofloxacin, resistance levels are rising with increased usage. While NF susceptibility is historically stable, TMP-SMX resistance is slightly higher in children compared to adults. In both adults and children, antimicrobial resistance patterns vary according to clinical practice site, with ambulatory urology patients showing the highest resistance. This suggests that physician's prescribing patterns contribute to antimicrobial resistance.
\end{abstract}

Keywords: ciprofloxacin; trimethoprim-sulfamethoxazole; nitrofurantoin; susceptibility; bacterial resistance; E. coli; urinary cultures; urinary tract infection 


\section{Introduction}

Antimicrobial drug resistance is a major concern in medicine. Urinary tract infections (UTIs) are common in both adults and children and are usually treated empirically. As a result, antimicrobial susceptibility is important for guiding therapy selection. Since susceptibility trends vary among countries, hospitals, clinical practice sites (ambulatory versus hospital), and patient groups (adult versus child), understanding these trends is important to infection management. While microbial resistance may be innate or acquired from spontaneous or induced mutation, contributions of physician usage patterns on selective pressures are unclear. To better understand this association, we investigated patterns of urinary Escherichia coli (E. coli) antimicrobial susceptibility within the same institution in different medical specialty practice sites, with the assumption that these sites reflect elements of physician specialists' usage patterns. Since ciprofloxacin (CP) usage is limited in children, we compared CP resistance in adults and children to two other commonly used antimicrobial agents: trimethoprim-sulfamethoxazole (TMP-SMX) and nitrofurantoin (NF).

\section{Results}

\subsection{Urinary Cultures}

A total of 156,730 urinary cultures were examined. Of these, 38,890 (24.8\%) were pediatric cultures (annual average of $3889 \pm 230$ [SD, Standard deviation]) and 117,840 (75.2\%) were adult (annually 11,784 \pm 1752 ). Of these, 7,414 cultures (19.1\%) were positive for $E$. coli from the pediatric hospital and 26,582 from adult cultures $(22.6 \%)$. The majority of these were from females: 5979 $(80.2 \pm 3.8 \%)$ from girls, and 22,950 (86.7 $\pm 1.6 \%)$ from adult females (Table 1$)$. Most urinary specimens positive for $E$. coli were from the ambulatory outpatient clinic and distributed as shown (Table 2).

Table 1. Patient demographics $(\mathrm{N}=$ number$)$.

\begin{tabular}{ccccc}
\hline Group & Pediatric N & $\mathbf{\%}$ & Adult N & \% \\
\hline Total positive urine culture & 38,890 & 24.8 & 117,840 & 75.2 \\
E. coli positive & 7414 & 19.1 & 26,582 & 22.6 \\
Females & 5979 & 80.2 & 22,950 & 86.7 \\
Males & 1416 & 19.8 & 3433 & 13.3 \\
Unknown gender & 19 & 0.2 & 199 & 0.8 \\
\hline
\end{tabular}

Table 2. E. coli positive urinary cultures by clinical site $(\mathrm{N}=$ number, OPT $=$ outpatient, $\mathrm{INPT}=$ inpatient, ED = emergency department, Gen Peds = general pediatrics, Gen Med = general medicine, OB = obstetrics, GYN = gynecology, $\mathrm{URO}=$ urology).

\begin{tabular}{ccccc}
\hline Group & Pediatric N & \% & Adult N & \% \\
\hline All Treatment Sites & 7414 & 19.1 & 26,582 & 22.6 \\
OPT & 6245 & 84.2 & 22,868 & 86 \\
INPT & 639 & 8.6 & 3709 & 14 \\
ED & 1719 & 23.2 & 5444 & 20.5 \\
Gen Peds/Gen Med & 1267 & 17.1 & 12,616 & 47.5 \\
OB/GYN & 698 & 9.4 & 917 & 3.4 \\
URO & 283 & 3.8 & 1703 & 6.4 \\
\hline
\end{tabular}




\subsection{Distribution of Antibiotics Susceptibilities}

Urinary E. coli was more likely to be susceptible to ciprofloxacin (CP) in children compared to adults $(p<0.002)$. Both child and adult sites showed decreasing susceptibilities from 100\% susceptibility in 2002 to $90 \%$ in 2011 in children, as compared to 74\% in adults (Figure 1). Outpatient (OPT) urine cultures had higher susceptibility to CP than inpatient specimen in both children and adults, with a larger difference in adults (Figure 2). Susceptibility also varied among ambulatory sites, with the lowest susceptibilities in the Urology clinic specimens (73\% in children) as compared to other sites that remained between 88 and 95\%. Adult urology ambulatory specimens showed a drop in susceptibility to $55 \%$ by 2011 (Tables 3 and 4 and Figure 3a). Similarly by 2011 inpatient hospital specimens were only $60 \%$ susceptible to ciprofloxacin (Table 4 and Figure $3 b$ ). All $p$-values were $<0.02$.

Figure 1. Adult and pediatric ciprofloxacin susceptibility trends for E. coli positive urinary cultures.

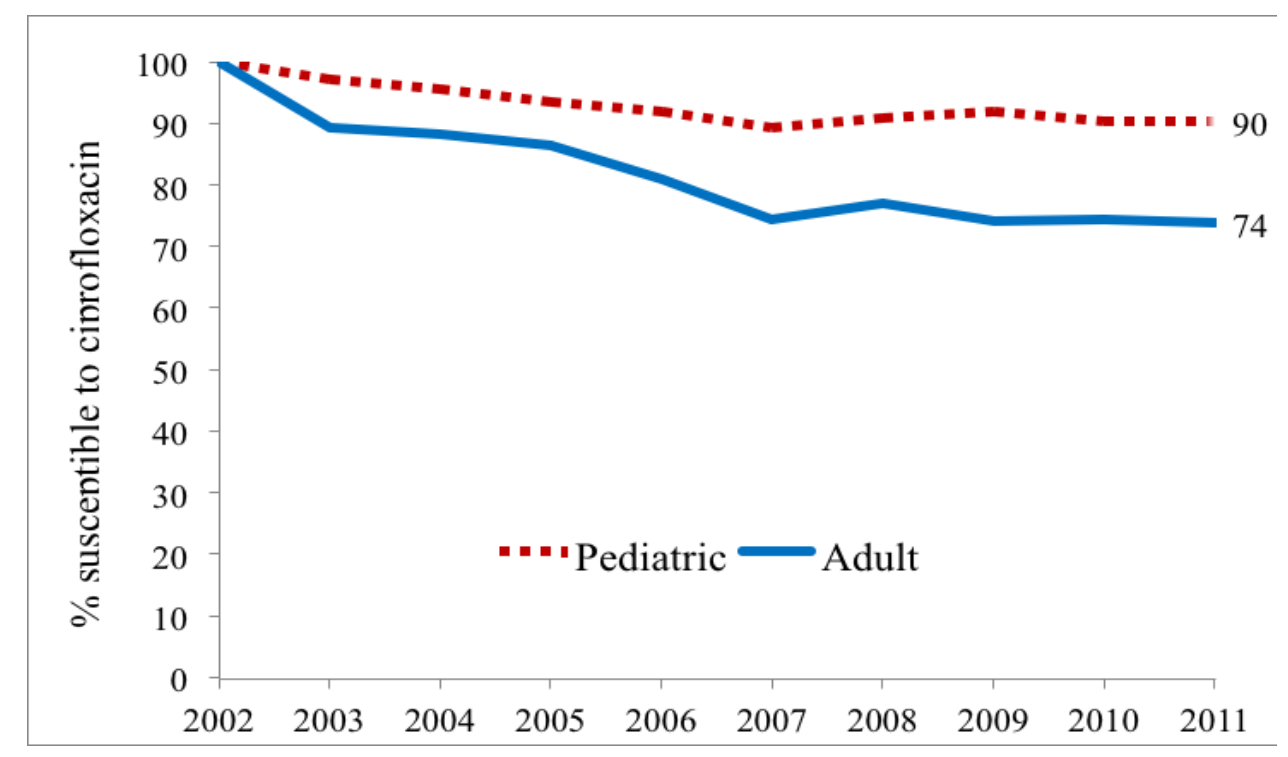

Figure 2. Outpatient and inpatient adult and pediatric ciprofloxacin susceptibility trends for E. coli positive urinary cultures $(\mathrm{OPT}=$ outpatient, $\mathrm{INPT}=$ inpatient $)$.

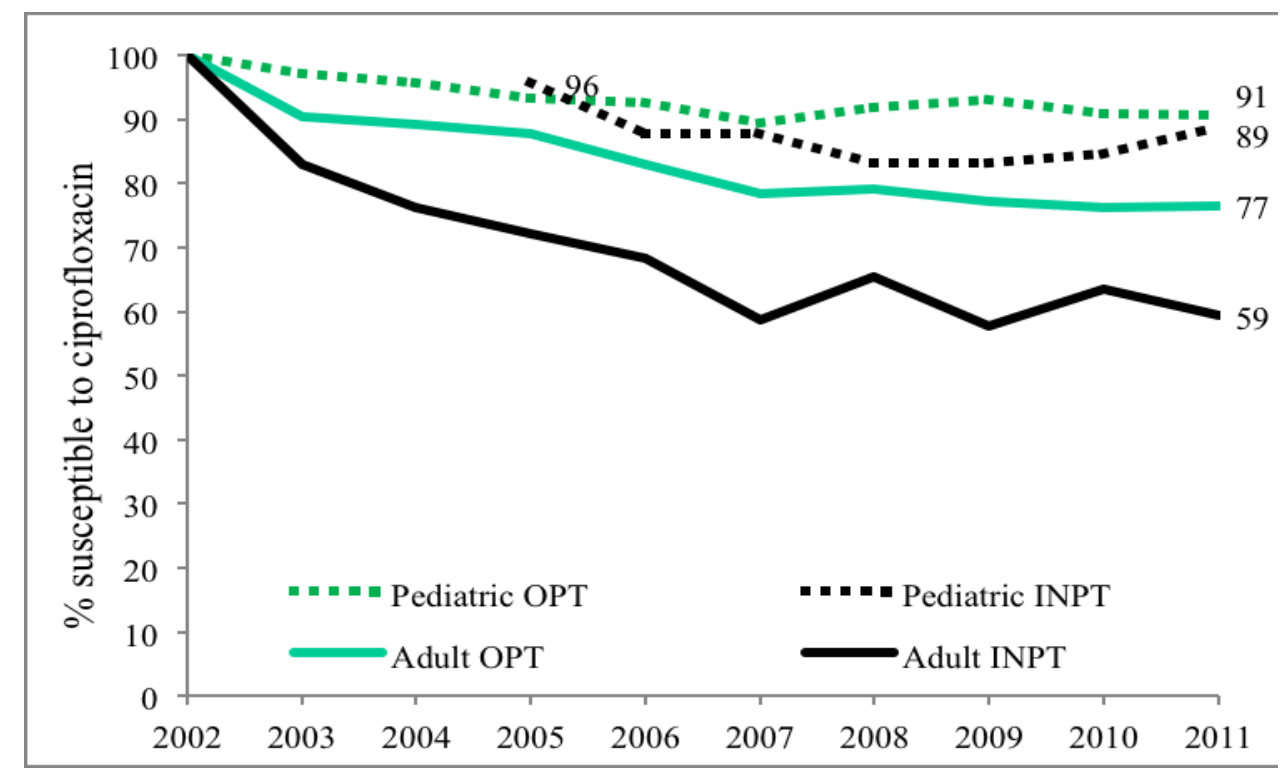


Table 3. Ciprofloxacin susceptibilities of pediatric E. coli positive urinary cultures by site $(\mathrm{N}=$ number, OPT = outpatient, INPT = inpatient, $\mathrm{ED}=$ emergency department, Gen Peds $=$ general pediatrics, $\mathrm{OB}=$ obstetrics, $\mathrm{URO}=$ urology).

\begin{tabular}{ccccccccc}
\hline Year & $\begin{array}{c}\text { All } \boldsymbol{E} \text {. coli } \\
\text { Cultures N }\end{array}$ & $\begin{array}{c}\text { All Sites } \\
\text { N (\%) }\end{array}$ & $\begin{array}{c}\text { OPT } \\
\text { N (\%) }\end{array}$ & $\begin{array}{c}\text { INPT } \\
\text { N (\%) }\end{array}$ & $\begin{array}{c}\text { ED } \\
\text { N (\%) }\end{array}$ & $\begin{array}{c}\text { Gen Peds } \\
\text { N (\%) }\end{array}$ & OB N (\%) & URO N (\%) \\
\hline 2002 & 528 & $490(100)$ & N/A & N/A & $93(100)$ & N/A & N/A & N/A \\
2003 & 577 & $519(97)$ & N/A & N/A & $109(100)$ & N/A & N/A & N/A \\
2004 & 1074 & $861(95.5)$ & N/A & N/A & $113(99.1)$ & N/A & N/A & N/A \\
2005 & 1144 & $968(93.5)$ & $876(93.4)$ & $90(95.7)$ & $114(95)$ & $499(95.6)$ & $94(94)$ & $20(69)$ \\
2006 & 680 & $584(92)$ & $526(92.4)$ & $58(87.9)$ & $132(95.7)$ & $146(94.2)$ & $94(94.9)$ & $29(85.3)$ \\
2007 & 632 & $520(89.2)$ & $448(89.4)$ & $72(87.8)$ & $141(97.2)$ & $112(94.9)$ & $88(89.8)$ & $32(84.2)$ \\
2008 & 631 & $518(90.9)$ & $464(91.9)$ & $54(83.1)$ & $162(93.1)$ & $103(95.4)$ & $93(95.9)$ & $18(81.8)$ \\
2009 & 749 & $623(92)$ & $563(93.1)$ & $60(83.3)$ & $255(96.2)$ & $85(95.5)$ & $78(95.1)$ & $29(87.9)$ \\
2010 & 731 & $571(90.2)$ & $510(90.9)$ & $61(84.7)$ & $224(98.2)$ & $74(100)$ & $93(96.9)$ & $29(76.3)$ \\
2011 & 668 & $554(90.4)$ & $468(90.7)$ & $87(88.8)$ & $189(95.5)$ & $94(93.1)$ & $80(95.2)$ & $27(73)$ \\
Total & 7414 & 6208 & 5718 & 489 & 1532 & 1482 & 620 & 198 \\
Mean & 741 & $621(93)$ & $572(93)$ & $61(87)$ & $153(97)$ & $185(96)$ & $89(95)$ & $20(80)$ \\
SD & 205 & $162(3)$ & $158(3)$ & $26(4)$ & $54(2)$ & $159(2)$ & $7(2)$ & $11(7)$ \\
\hline
\end{tabular}

Table 4. Ciprofloxacin susceptibilities of adult E. coli positive urinary cultures by site $(\mathrm{N}=$ number, $\mathrm{OPT}=$ outpatient, $\mathrm{INPT}=$ inpatient, $\mathrm{ED}=$ emergency department, Gen Med = general medicine, GYN = gynecology, $\mathrm{URO}=$ urology).

\begin{tabular}{ccccccccc}
\hline Year & $\begin{array}{c}\text { All } \boldsymbol{E} \text { c coli } \\
\text { Cultures N }\end{array}$ & $\begin{array}{c}\text { All Sites } \\
\text { N (\%) }\end{array}$ & $\begin{array}{c}\text { OPT } \\
\text { N (\%) }\end{array}$ & $\begin{array}{c}\text { INPT } \\
\text { N (\%) }\end{array}$ & $\begin{array}{c}\text { EDN } \\
(\%)\end{array}$ & $\begin{array}{c}\text { Gen Med } \\
\text { N (\%) }\end{array}$ & $\begin{array}{c}\text { GYN } \\
\text { N (\%) }\end{array}$ & URO \\
\hline 2002 & 1557 & $1306(100)$ & $1083(100)$ & $223(100)$ & $268(100)$ & $470(100)$ & $70(100)$ & $123(100)$ \\
2003 & 1538 & $1233(89.2)$ & $1061(90.3)$ & $168(82.8)$ & $333(88.8)$ & $501(96.7)$ & $44(89.8)$ & $101(77.7)$ \\
2004 & 3484 & $2683(88.2)$ & $2500(89.2)$ & $183(76.3)$ & $344(88.4)$ & $1953(91.8)$ & $30(83.3)$ & $104(68.9)$ \\
2005 & 4456 & $3518(86.4)$ & $3270(87.7)$ & $248(72.1)$ & $338(81.3)$ & $2700(90.4)$ & $50(87.7)$ & $97(68.3)$ \\
2006 & 2425 & $1817(81)$ & $1599(83.1)$ & $218(68.3)$ & $376(78.7)$ & $949(89.6)$ & $48(85.7)$ & $96(64.9)$ \\
2007 & 2298 & $1589(74.5)$ & $1345(78.3)$ & $244(58.7)$ & $367(77.1)$ & $722(87.8)$ & $62(80.5)$ & $98(58.7)$ \\
2008 & 2540 & $1816(76.9)$ & $1571(79.1)$ & $245(65.5)$ & $454(77.5)$ & $825(88)$ & $67(73.6)$ & $89(55.3)$ \\
2009 & 2702 & $1852(74.1)$ & $1631(77.1)$ & $221(57.7)$ & $470(68.5)$ & $841(90.3)$ & $79(77.5)$ & $96(61.5)$ \\
2010 & 2425 & $1847(74.3)$ & $1621(76.1)$ & $226(63.5)$ & $466(74.1)$ & $789(85.2)$ & $116(88.5)$ & $71(47)$ \\
2011 & 2857 & $1955(74)$ & $1712(76.6)$ & $239(59.5)$ & $499(72.1)$ & $770(85.9)$ & $141(87.6$ & $92(55.4)$ \\
Total & 26582 & 19616 & 17396 & 2216 & 3715 & 10520 & 707 & 967 \\
Mean & 2658 & $1962(82)$ & $1740(84)$ & $222(70)$ & $392(81)$ & $1052(91)$ & $71(85)$ & $97(66)$ \\
SD & 858 & $676(9)$ & $670(8)$ & $27(13)$ & $76(9)$ & $710(5)$ & $34(7)$ & $13(15)$ \\
\hline
\end{tabular}


Figure 3. (a) Outpatient pediatric ciprofloxacin susceptibility trends for E. coli positive urinary cultures $(\mathrm{ED}=$ emergency department, All Gen Peds clinics = general pediatrics clinics, $\mathrm{OB}=$ Obstetrics clinics/Labor and delivery, URO = urology clinics); (b) Outpatient adult ciprofloxacin susceptibility trends for E. coli positive urinary cultures (Students' health $=$ undergraduate and graduate students' clinic, ED = emergency department, All med clinics $=$ general medicine clinics, GYN = gynecology clinics, URO = urology clinics).

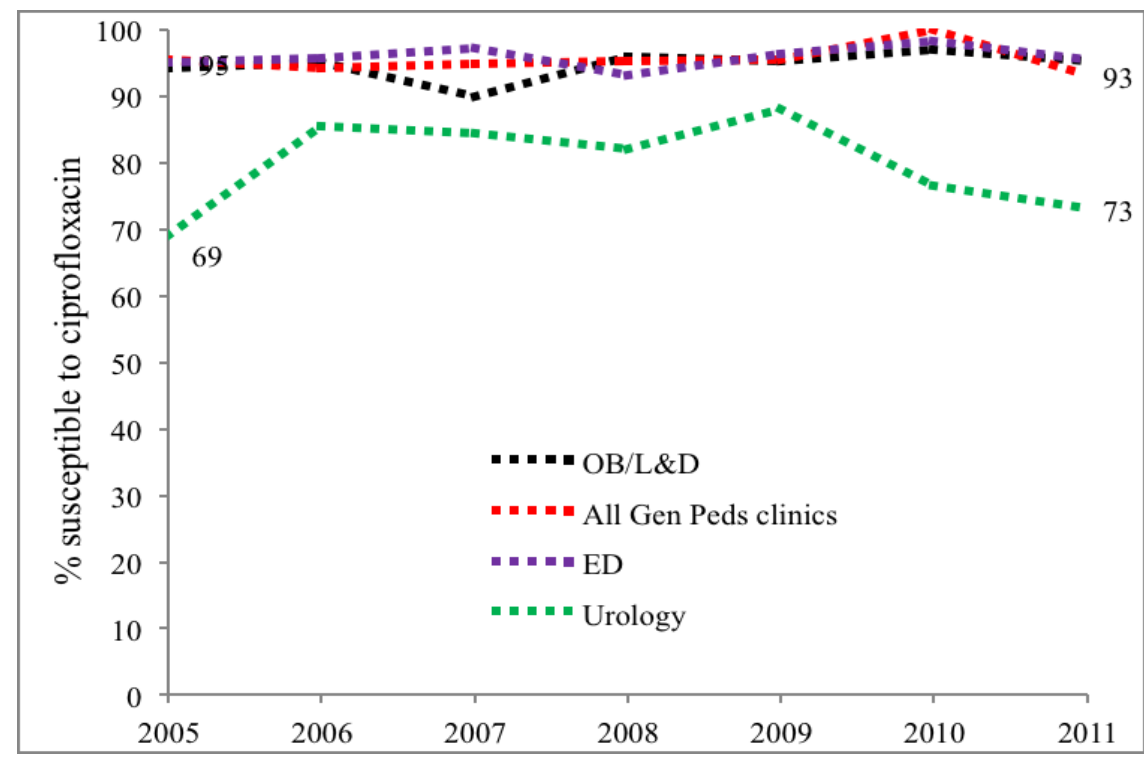

(a)

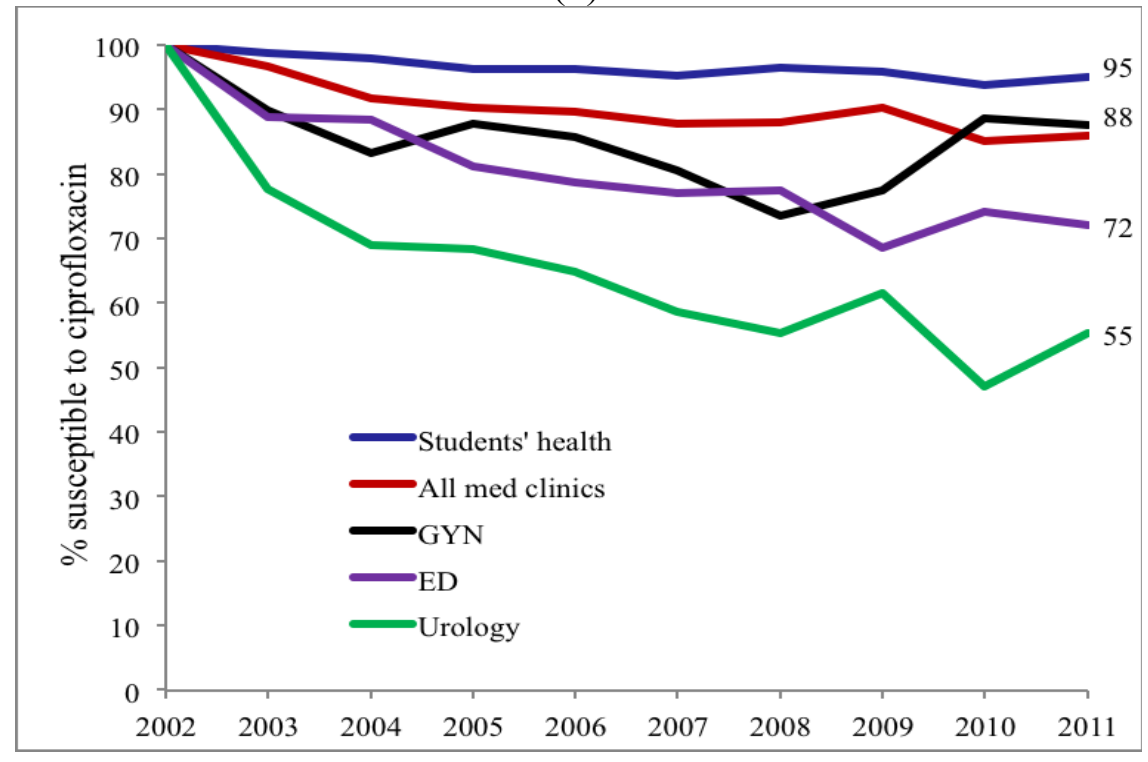

(b)

Trimethoprim-sulfamethoxazole (TMP-SMX) susceptibility is lower in children than adults. While TMP-SMX susceptibility declined during the study period, the rate of decrease was lower than for CP. Again, susceptibility varied with clinical treatment site and lowest susceptibility occurred in pediatric and adult urology ambulatory sites (Figure 4a,b, $p<0.02$ ). E. coli NF susceptibility for both children and adult specimens was, meanwhile, relatively stable varying from 93\%-100\% between 2002 and 2011 without significant difference between adults and children nor among treatment sites. Comparison of these three commonly used antibiotics within ambulatory urology demonstrated three 
patterns of E. coli susceptibility: (a) no differences in NF susceptibility between children and adults; (b) lower TMP-SMX susceptibilities in children than adults; and (c) higher CP susceptibility in children than adults Figures 2-5).

Figure 4. (a) Outpatient pediatric TMP-SMX susceptibility trends for E. coli positive urinary cultures $(E D=$ emergency department, All Gen Peds clinics = general pediatrics clinics, $\mathrm{OB}=$ obstetrics clinics/Labor and delivery, $\mathrm{URO}=$ urology clinics); (b) Outpatient adult TMP-SMX susceptibility trends for E. coli positive urinary cultures (Students' health = undergraduate and graduate students' clinic, ED = emergency department, All med clinics = general medicine clinics, GYN = gynecology clinics, URO = urology clinics).

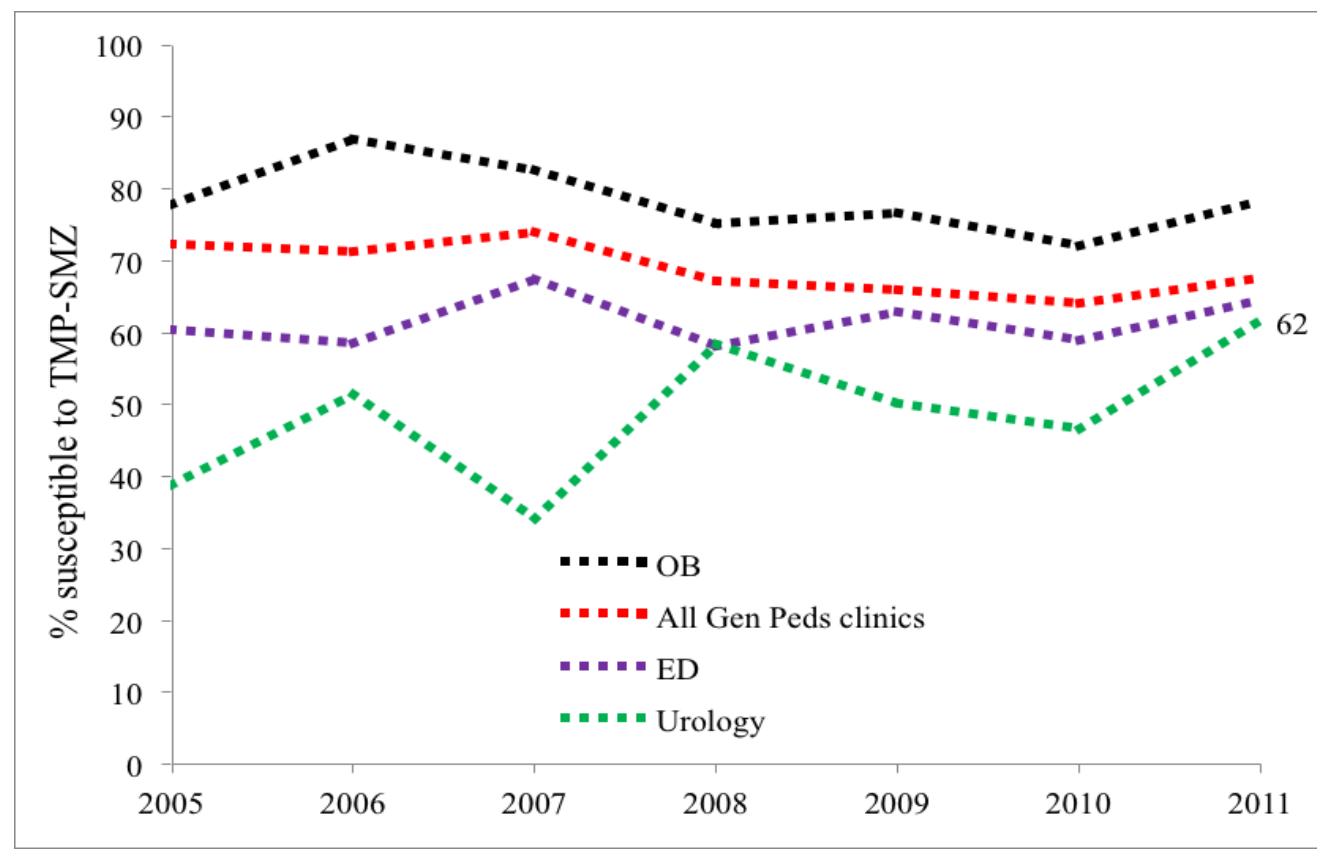

(a)

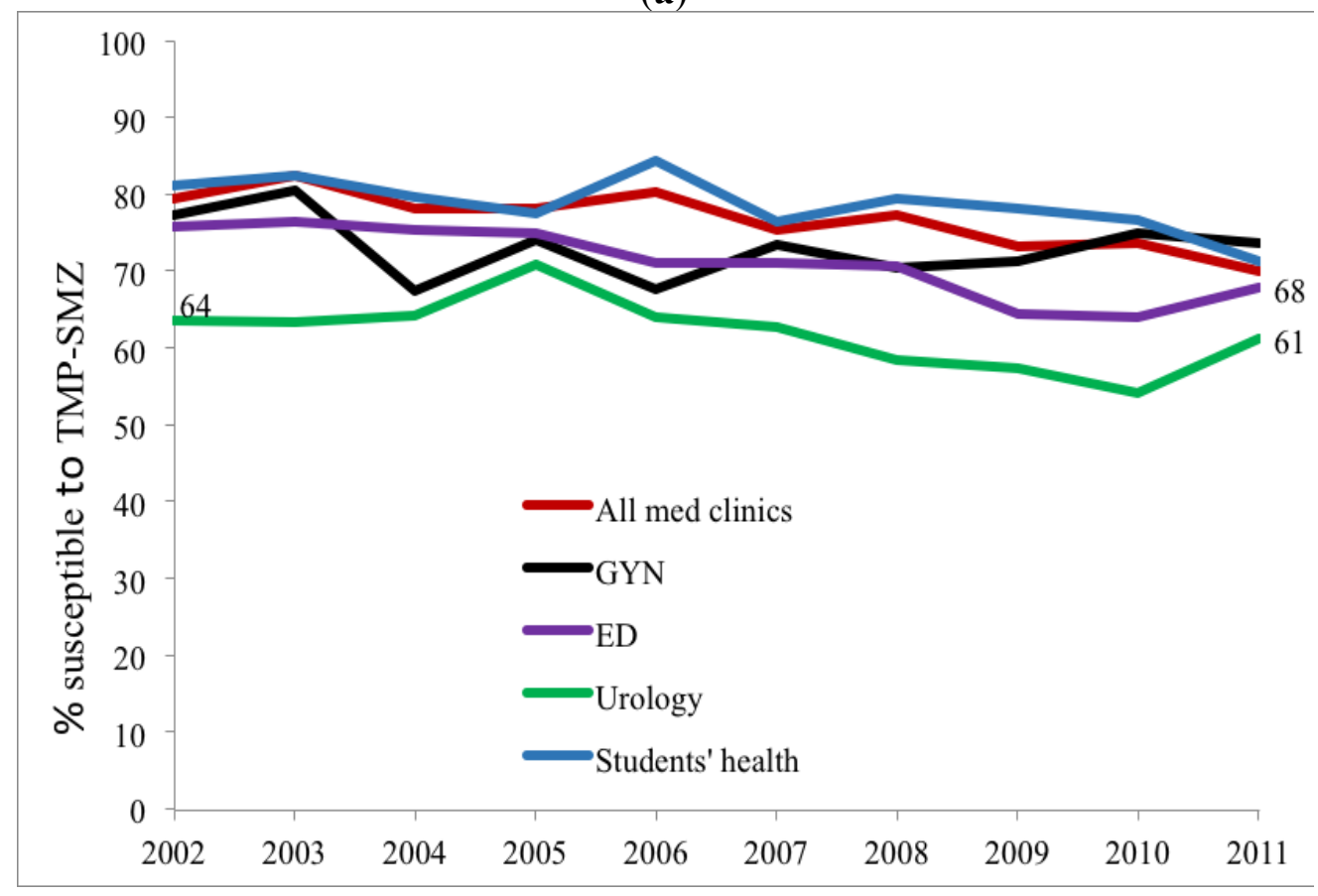

(b) 
Figure 5. (a) Pediatric urology clinic susceptibility trends for three commonly prescribed antimicrobial agents for $E$. coli positive urinary cultures $(\mathrm{NF}=$ nitrofurantoin, $\mathrm{CP}=$ ciprofloxacin, TMP-SMX = trimethoprim-sulfamethoxazole); (b) Adult urology clinic susceptibility trends for three commonly prescribed antimicrobial agents for E. coli positive urinary cultures $(\mathrm{NF}=$ nitrofurantoin, $\mathrm{CP}=$ ciprofloxacin, $\mathrm{TMP}-\mathrm{SMX}=$ trimethoprim-sulfamethoxazole).

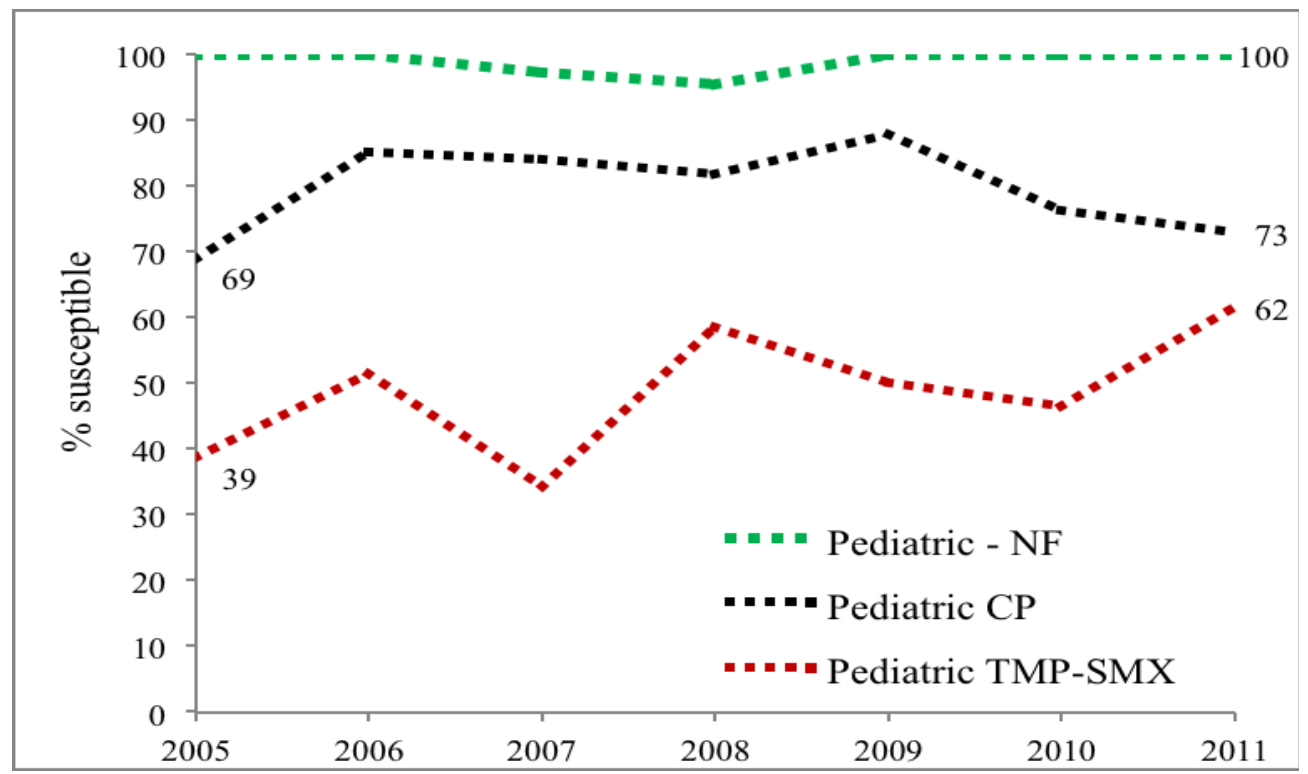

(a)

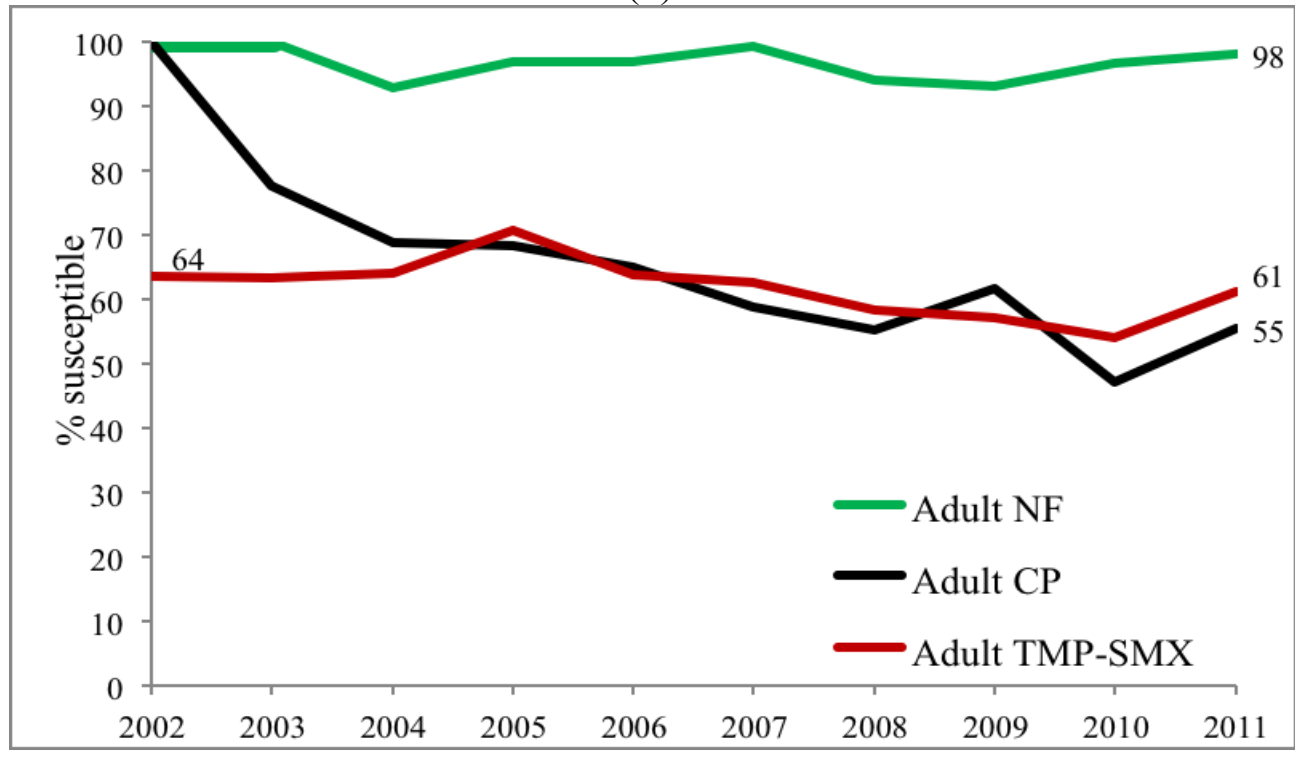

(b)

\section{Discussion}

Simultaneous to development of powerful and less toxic antimicrobial agents, infecting organisms are developing more resistance to antimicrobial agents. This has been a trend that has been reported over the last 20 years, as widespread usage of antimicrobial agents is believed to create selection pressure for more resistant organisms [1]. Case-control studies comparing children with and without TMP-SMX resistant organisms have shown that children who had antimicrobials agents for greater 
than four weeks in the previous six months were 23 times more likely to have resistance compared with those without resistance; children with genitourinary abnormalities were 2.4 times more likely to have resistance; and children with previous hospital admissions 2.3 times more likely [2] In other cases, specific clones of multidrug-resistant E. coli have been shown to behave with "epidemic outbreak" characteristics. The sources of these clonal epidemic-like outbreaks are unclear; some evidence implicates food and/or contact with food sources that had antimicrobial agent contact [3]. While international, national, regional, local, or hospital-wide antibiograms reflecting organism resistance attract attention [4,5], there is limited research on organism resistance and susceptibility diversity by practice site within institutions.

In this study we document that: (1) E. coli antimicrobial susceptibility differs widely between adults and children; (2) further variation occurs by practice site within a single medical center; and (3) differing drug trends in susceptibilities may occur. We hypothesize that these practice site susceptibility differences reflect the differing antimicrobial practice patterns of specialists and their specialty practice patterns. These antimicrobial drug trends may show: (a) differing susceptibilities between adults and children; (b) similar decreasing antimicrobial drug susceptibilities for both adults and children; and (c) persistently low susceptibilities for both. Our findings suggest that antimicrobial susceptibility and resistance patterns are affected by specialty practice patterns.

This study shows that pediatric and adult $E$. coli UTI susceptibility trends differ for three commonly used antimicrobial agents. With limited usage in children overall CP susceptibilities (69\%-88\%) are high in children except for the lower levels in ambulatory urology with higher usage; in contrast results show lower susceptibility in adults with lowest levels in adult ambulatory urology. A decreasing susceptibility rate for E. coli in urology practices has been noted by others in Japan [6]. The widespread usage of TMP-SMX in children for otitis media in addition to UTI is reflected in overall E. coli susceptibilities of $39 \%-62 \%$ while adult susceptibilities range from $61 \%-71 \%$. The stable higher $E$. coli susceptibilities of NF for both adults $(>92 \%)$ and children $(>95 \%)$ for the study periods most likely reflect relatively limited usage of NF for lower urinary tract infections and prophylaxis as reported by others [7]. By combining data from multiple institutional and internal practice sites, others have not revealed the range of $E$. coli susceptibilities within a single site nor the differences between children and adults [8]. Our data show that using individual hospital or institutional antibiograms to guide empiric antimicrobial treatment of a UTI in child or adult, moreover, may be misleading (Figures 1 and 2 and Tables 3 and 4).

These data and conclusions are limited by several considerations. First, prior to 2005 the sources of our pediatric specimens were not separated by outpatient and inpatient locations, so data trends are from 2005 forward, and second, CP patterns were not recorded in 2002 for technical reasons. Because data were de-identified, we could not eliminate repeat specimen data for patients. While multiple specimens from individuals could decrease or increase our susceptibility calculations, we believe that the large number of specimens involved reflect valid trends. Our usage of threshold of $10,000 \mathrm{CFU} / \mathrm{ml}$ as a positive culture may have excluded some urinary specimens with fewer resistant organisms, and this could overestimate our susceptibility reports. Finally, these studies compare data from an institution that offers primary medical care to the surrounding community in addition to regional and national tertiary care, thus these data may be unique based upon this patient population. 


\section{Experimental}

After Stanford University Medical Center Institutional Review Board approval was obtained for this study, we performed a retrospective review of all urine cultures submitted to Stanford University Medical Center (SUMC) Microbiology Laboratory from 2002 to 2011. Cultures were from Stanford University Hospitals and Clinics (SUH) reflecting specimens obtained primarily from adults, and from Lucile Packard Children's Hospital and Clinics (LPCH) from children and adult women seen in obstetrics clinic (OB) or admitted to labor and delivery (L\&D). Data included inpatient (INPT) and outpatient (OPT) urinary specimens obtained by all collection methods; repeat specimens per individual could not be excluded because data were de-identified. Microbiological data were obtained from two sources: (1) the SUMC clinical microbiology laboratory electronic database that contains urinary culture and susceptibility reports and annual hospital-wide antimicrobial susceptibility data; and (2) the STRIDE database (Stanford Translational Research Integrated Database Environment that compiles annual urinary culture specimen numbers, including positive and negative results. This is an NIH (National Institutes of Health) sponsored program that compiles clinical data for research use from SUMC. From the annual microbiology laboratory reports, hospital-wide trends were compiled for both the adult and pediatric hospitals ("antibiograms"). These antibiogram reports include cultures and susceptibilities from any type of culture (example, urine, blood, tracheal aspirates, etc.) processed at the SUMC laboratory.

STRIDE total urine cultures data were screened for selection using UTI diagnosis based on the following ICD-9 codes: 599.0 (UTI site not specified), 595 (cystitis), 590.1 (Acute pyelonephritis), 590.8 (Pyelonephritis unspecified). These data were segregated between the adult and pediatric hospitals by using an age cutoff of 18 years and below for pediatric patient data. This method generated total numbers of urinary cultures sent for populations. Antibiotic urinary susceptibility data was retrieved from the microbiology laboratory database that stores data for adult and pediatric hospitals separately. All SUMC clinical microbiology laboratory electronic urinary culture data were first parsed for overall antimicrobial susceptibility trends, then separated between INPT and OPT. The OPT clinical site data were further subdivided to various ambulatory clinics. For the adult hospital the OPT sites included the ED (emergency department), Gen Med (all internal medicine clinics including Stanford family medicine, Stanford medical group, Stanford family medicine, off-site community practice medical clinics using the Stanford laboratory), GYN (Gynecology) and URO (Urology). The pediatric OPT sites included the ED, Gen Peds (General pediatric clinics, including onsite and offsite community practice groups), OB (Obstetrics/Labor and delivery) and URO (Urology). Of note, except for the ED, prior to 2005, the pediatric hospital data entry did not code separately for outpatient specialty clinic sites.

Urinary specimens with $E$. coli were considered positive at a threshold of $10,000 \mathrm{cfu} / \mathrm{mL}$ (colony forming units per milliliter) in purity or predominance. This threshold was chosen because antimicrobial susceptibilities are routinely reported for $10,000 \mathrm{CFU} / \mathrm{mL}$ and we were examining $E$. coli susceptibilities in urinary cultures not urinary tract infection rates. Antimicrobial susceptibilities were performed using a Vitek2 microbial identification system (bioMérerieux Inc., Durham, North Carolina, USA) and MICs were interpreted according to the Clinical and Laboratory Standard Institute M100 document. 


\section{Statistical Analysis}

We calculated the percentage annual urinary culture susceptibility as the numbers of specimens sensitive to the specific antibiotic divided by the annual E. coli positive cultures (CP, TMP-SMX and NF). The figures obtained were plotted annually to obtain the annual trend corresponding to the various clinical sites evaluated. Independent (unpaired) two-tailed Student's $t$-test ( $p$-value) were calculated for comparison of adult versus pediatric sites susceptibility to assess the statistical significance of differences seen among clinical sites in adults and children.

\section{Conclusions}

We report that antimicrobial susceptibilities for $E$. coli urinary cultures from a single institution may differ significantly between adults and children for three commonly used agents. Differences may occur, furthermore, among various practice sites within a single hospital site. We suggest that these differences in susceptibility reflect distinct specialty clinical prescribing practices. As a result of these variations, reliance upon any single regional or local antibiograms within large practice medical centers could be misleading.

\section{Author Contributions}

Winifred Owumi designed the study, performed data retrieval and analyses, and wrote portions of the paper. Niaz Banaei directed and reviewed data retrieval and reviewed the paper. Linda D. Shortliffe designed the study, directed analyses, and wrote and edited the paper.

\section{Conflicts of Interest}

The authors declare no conflict of interest.

\section{References}

1. Kunin, C.M. Resistance to antimicrobial drugs-A worldwide calamity. Ann. Intern. Med. 1993, 118, 557-561.

2. Allen, U.D.; MacDonald, N.; Fuite, L.; Chan, F.; Stephens, D. Risk factors for resistance to "first-line" antimicrobials among urinary tract isolates of Escherichia coli in children. Can. Med. Assoc. J. 1999, 160, 1436-1440.

3. Drexler, M. Secret Agents: The Menace of Emerging Infections; Joseph Henry Press: Washington, DC, USA, 2002.

4. Morrissey, I.; Hackel, M.; Badal, R.; Bouchillon, S.; Hawser, S.; Biedenbach, D. A review of ten years of the study for monitoring antimicrobial resistance trends (SMART) from 2002 to 2011. Pharmaceuticals 2013, 6, 1335-1346.

5. Bouchillon, S.K.; Badal, R.E.; Hoban, D.J.; Hawser, S.P. Antimicrobial susceptibility of inpatient urinary tract isolates of gram-negative bacilli in the United States: Results from the study for monitoring antimicrobial resistance trends (SMART) program: 2009-2011. Clin. Ther. 2013, 35, 872-877. 
6. Yamamichi, F.; Shigemura, K.; Matsumoto, M.; Nakano, Y.; Tanaka, K.; Arakawa, S.; Fujisawa, M. Relationship between urinary tract infection categorization and pathogens' antimicrobial susceptibilities. Urol. Int. 2012, 88, 198-208.

7. Kashanian, J.; Hakimian, P.; Blute, M., Jr.; Wong, J.; Khanna, H.; Wise, G.; Shabsigh, R. Nitrofurantoin: The return of an old friend in the wake of growing resistance. BJU Int. 2008, 102, 1634-1637.

8. McGregor, J.C.; Elman, M.R.; Bearden, D.T.; Smith, D.H. Sex- and age-specific trends in antibiotic resistance patterns of Escherichia coli urinary isolates from outpatients. BMC Fam. Pract. 2013, 14, e25.

(C) 2014 by the authors; licensee MDPI, Basel, Switzerland. This article is an open access article distributed under the terms and conditions of the Creative Commons Attribution license (http://creativecommons.org/licenses/by/3.0/). 